Portland State University

PDXScholar

Mechanical and Materials Engineering Faculty

Publications and Presentations

$12-10-2013$

\title{
Capillary Channel Flow Experiments Aboard the International Space Station
}

\author{
Michael Conrath \\ University of Bremen \\ P. J. Canfield \\ University of Bremen \\ P. M. Bronowicki \\ University of Bremen \\ Michael E. Dreyer \\ University of Bremen \\ Mark M. Weislogel \\ Portland State University, weisloge@pdx.edu
}

See next page for additional authors

Follow this and additional works at: https://pdxscholar.library.pdx.edu/mengin_fac

Part of the Fluid Dynamics Commons

Let us know how access to this document benefits you.

\section{Citation Details}

Conrath, M., Canfield, P. J., Bronowicki, P. M., Dreyer, M. E., Weislogel, M. M., \& Grah, A. (2013). Capillary channel flow experiments aboard the International Space Station. Physical Review E, 88(6), 063009.

This Article is brought to you for free and open access. It has been accepted for inclusion in Mechanical and Materials Engineering Faculty Publications and Presentations by an authorized administrator of PDXScholar. Please contact us if we can make this document more accessible: pdxscholar@pdx.edu. 
Authors

Michael Conrath, P. J. Canfield, P. M. Bronowicki, Michael E. Dreyer, Mark M. Weislogel, and A. Grah 


\title{
Capillary channel flow experiments aboard the International Space Station
}

\author{
M. Conrath, ${ }^{1}$ P. J. Canfield, ${ }^{1}$ P. M. Bronowicki, ${ }^{1}$ M. E. Dreyer,,${ }^{1,}$ M. M. Weislogel, ${ }^{2}$ and A. $\mathrm{Grah}^{3}$ \\ ${ }^{1}$ Center of Applied Space Technology and Microgravity (ZARM), University of Bremen, Am Fallturm 01, 28359 Bremen, Germany \\ ${ }^{2}$ Department of Mechanical and Materials Engineering, Portland State University, PO Box 751, Portland, Oregon 97207, USA \\ ${ }^{3}$ European Commission, Institute for Energy and Transport, Petten, The Netherlands
}

(Received 8 May 2013; revised manuscript received 7 November 2013; published 10 December 2013)

\begin{abstract}
In the near-weightless environment of orbiting spacecraft capillary forces dominate interfacial flow phenomena over unearthly large length scales. In current experiments aboard the International Space Station, partially open channels are being investigated to determine critical flow rate-limiting conditions above which the free surface collapses ingesting bubbles. Without the natural passive phase separating qualities of buoyancy, such ingested bubbles can in turn wreak havoc on the fluid transport systems of spacecraft. The flow channels under investigation represent geometric families of conduits with applications to liquid propellant acquisition, thermal fluids circulation, and water processing for life support. Present and near future experiments focus on transient phenomena and conduit asymmetries allowing capillary forces to replace the role of gravity to perform passive phase separations. Terrestrial applications are noted where enhanced transport via direct liquid-gas contact is desired.
\end{abstract}

DOI: 10.1103/PhysRevE.88.063009

PACS number(s): 47.20.Dr, 47.35.Pq, 47.55.nb, 47.60.Dx

\section{INTRODUCTION}

Direct contact fluid transport equipment such as bubble columns, trickle beds, and cooling towers [1] inherently rely on buoyancy via gravitational acceleration. To perform such vital unit operations without the aid of gravity, we can instead rely on surface tension in microfluidic systems where the relative influence of gravity is dramatically reduced—as in the case of screen separators, wicks, membranes, capillary pipes, and other devices [2-4]. In the microgravity environment of many orbiting spacecraft the microfluidic limit no longer applies and capillary-dominated systems are permissible that are 1000-fold larger than their terrestrial counterparts. In this article, ongoing experiments currently aboard the International Space Station (ISS) are reported concerning forced flows along partially open conduits. In contrast to similar terrestrial flows that are mainly dominated by viscous resistance [5], the ISS experiments are largely and uniquely inertia dominated, motivating in part our efforts to further explore them. It is found for such flows that an abrupt transition occurs between steady-state and unsteady "choked" flow conditions, leading to large surface deformations and periodic gas ingestion [6]. The instability mechanism is related to those of liquid jets [7] and rivulets [8-12].

\section{PREVIOUS INVESTIGATIONS}

Three representative capillary channel geometries that have received significant attention are depicted in Fig. 1. The means to study them in a microgravity ( $\mu \mathrm{g})$ environment have been (i) the drop tower Bremen with about $4.7 \mathrm{~s} \mu \mathrm{g}$ time, (ii) sounding rockets of the TEXUS program launched from Esrange near Kiruna in Sweden with about 6 min of $\mu \mathrm{g}$ time, and now (iii) the International Space Station ISS with long-term permanent $\mu \mathrm{g}$ time. The $\mu \mathrm{g}$ quality is approximately $10^{-5} g_{0}$ for both the drop tower and on ISS and $10^{-4} g_{0}$ aboard a sounding rocket.

*Corresponding author: michael.dreyer@zarm.uni-bremen.de
The parallel plate geometry of Fig. 1(a) was first studied in connection with capillary imbibition both on Earth and in microgravity [13-15]. Particularly in space, such open capillary channels are considered "liquid acquisition devices" since they may be exploited to passively imbibe and, hence, control large amounts of liquid over large distances in the near absence of gravity [16-18]. The channels may also be exploited to support forced flows at higher flow rates than are possible by wicking alone. Such was demonstrated in drop tower tests employing a variety of liquids and parallel plate channels to observe both capillary driven and forced flow behavior [19]. Sounding rocket experiments have been conducted to isolate the choking phenomena [20], expand the database [21,22], and add bubbly two-phase flows [23,24]. Progress of analytical and numerical model development for the parallel plate channel geometry has been reported $[21,22,25,26]$. The choking effect in " $U$ " -shaped grooves typified in Fig. 1(b) was examined using drop tower tests [27,28], and the triangular wedge geometry of Fig. 1(c) has been investigated both experimentally and numerically [29]. The unique opportunity to conduct such experiments in the long duration low-gravity environment aboard the ISS provides the ability to establish nearly ideal steady-state conditions. From such it is possible to detect the stability limit with greater accuracy, while significantly broadening the database for further theory development. The overall experiment is called the capillary channel flow (CCF) experiment and the present article reports an early look at a subset of the experimental data collected for steady flow in the parallel plate geometry for the unique parametric regimes possible in a laboratory aboard an orbiting spacecraft.

\section{THE CCF ISS EXPERIMENT}

The CCF experiment is a robotic ISS experiment commanded remotely from Earth. Astronaut involvement is required only for its installation and removal from the Microgravity Science Glovebox (MSG), which is located in the Destiny Module of the ISS [30]. The hardware functions are complicated by the unusual environment. The details of 


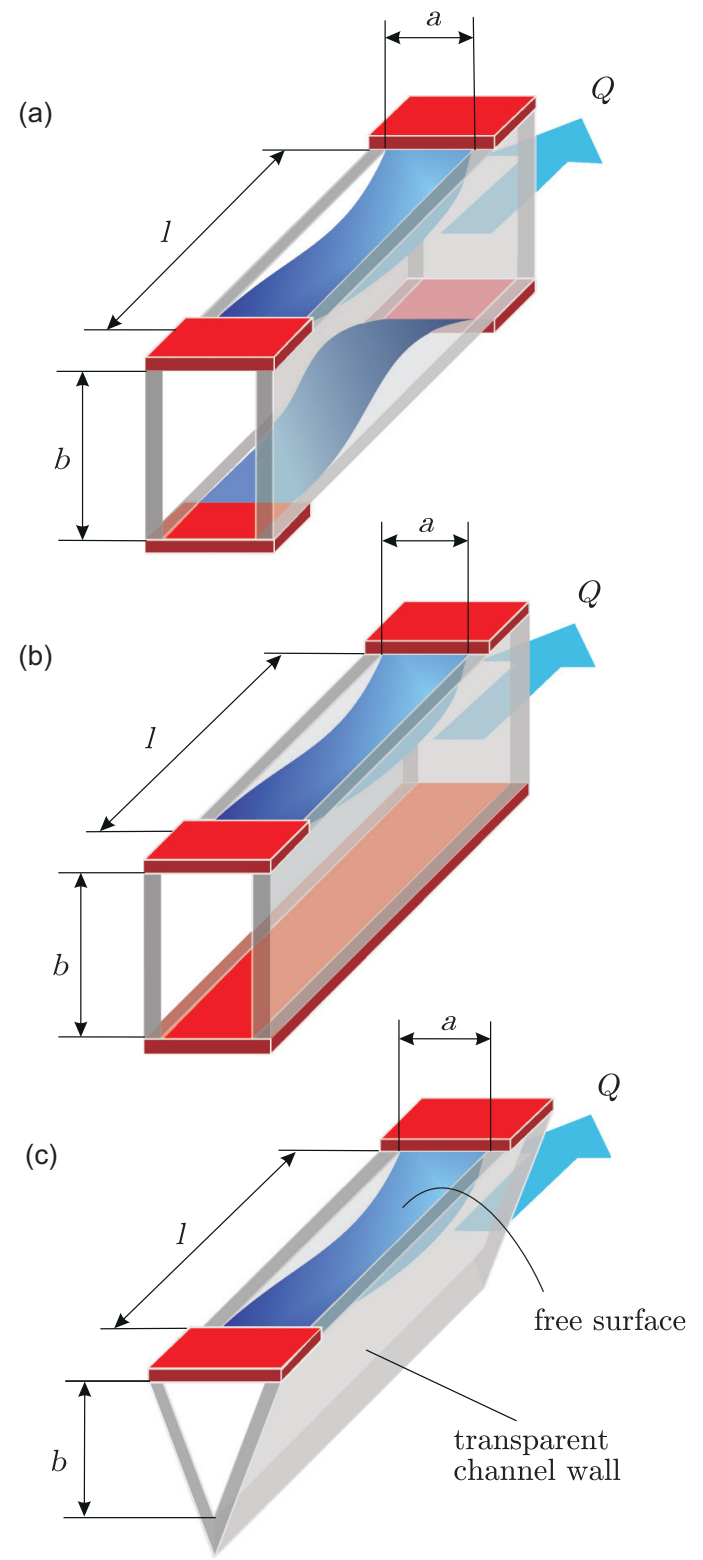

FIG. 1. (Color online) Capillary channel configurations that have been studied over the past decades. (a) Parallel plates, (b) groove, and (c) wedge. The channels have a width $a$, a height $b$, and a length of the free surface $l$. A pressure gradient between channel inlet and outlet is applied to generate a liquid flow with a flow rate $Q$.

setup, commanding, and control are described elsewhere [31]. Here we describe only the features of relevance to forced capillary channel flow between two parallel plates. Figure 2 displays the parallel plate configuration as seen from profile and cross-section views, respectively. The coordinate system and most relevant dimensions are labeled. The glass channel has width $a=5 \mathrm{~mm}$, height $b=25 \mathrm{~mm}$, and independently variable free surface length $l$ between 1 and $48 \mathrm{~mm}$ using two rigid sliding "lids" called sliders. The liquid is a hydrofluorether purchasable as HFE7500 and the ambient gas is nitrogen. At an ambient temperature of $(30 \pm 5){ }^{\circ} \mathrm{C}$, the liquid has a density $\rho=(1610 \pm 10) \mathrm{kg} / \mathrm{m}^{3}$, kinematic viscosity $v=(0.715 \pm 0.055) \times 10^{-6} \mathrm{~m}^{2} / \mathrm{s}$, and surface tension $\sigma=$ $(16.16 \pm 0.5) \mathrm{mN} / \mathrm{m}$. The liquid is perfectly wetting with zero
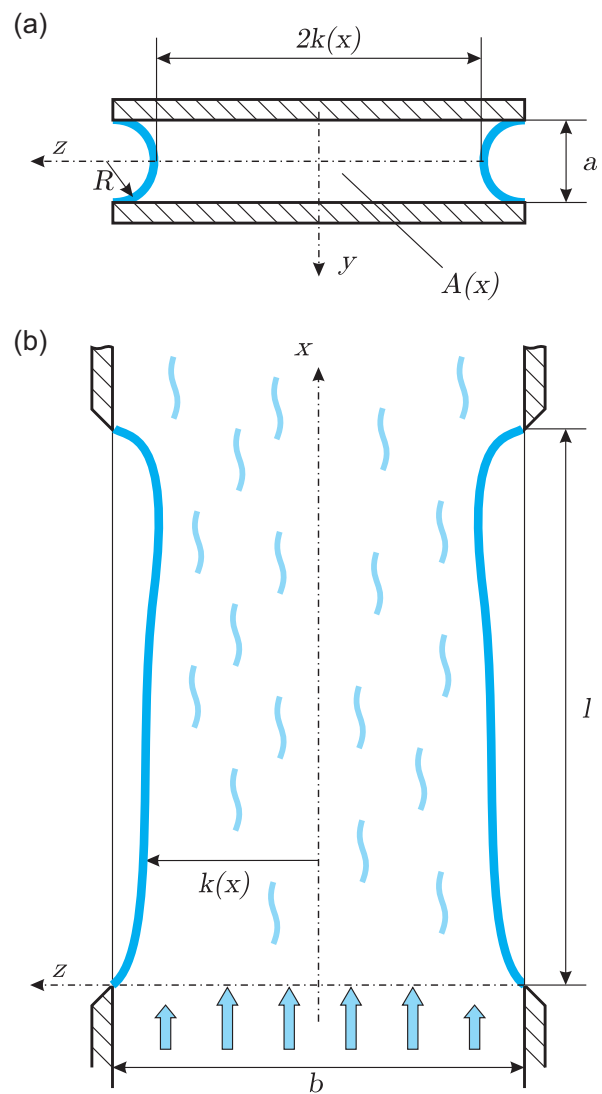

FIG. 2. (Color online) Parallel plate channel geometry: (a) $y z$ plane with the cross-sectional area of the flow $A(x)$ and the radius $R(x)$ at the surface. (b) $x z$ plane with the channel inlet at $x=0$ and the channel outlet at $x=l$ with free surface contour $k(x)$ (flow in the $x$ direction). The liquid pressure in the channel is lower than the outside pressure due to the curvature of the free surface.

degree contact angle along all walls. The liquid flow rate $Q$ is less than $20 \mathrm{ml} / \mathrm{s}$ and is driven by a pump. The pressure in the loop is determined primarily by the meniscus curvature in a compensation tube located near the inlet, as identified in Fig. 3. The absolute ambient pressure is $p_{a}=(105 \pm 10) \mathrm{kPa}$. The liquid accelerates from a reservoir through a converging nozzle and into the channel at Reynolds numbers less than 900. Thus, the flow possesses a developing velocity profile, the degree of which varies with flow rate and channel length. The free surface is pinned at the edges of both the inlet and outlet. At low flow rates the free surface also pins along the side edges of the channel, but as the flow rate increases the contact lines depin along these edges and are free to move inward along the channel walls. The profile $k(x)$ of the free surface along the channel is observed by high-speed video photography, as sketched in Fig. 2 and as recorded in Fig. 4.

\section{GENERAL EFFECTS}

Up to a critical flow rate, the surface pressure due to capillary curvature is able to withstand the inward suction forces caused by the liquid as it accelerates through the increasingly occluded passageway. In this steady subcritical regime, the free surface is only slightly deformed and remains stationary apart from minor oscillations originating at the 


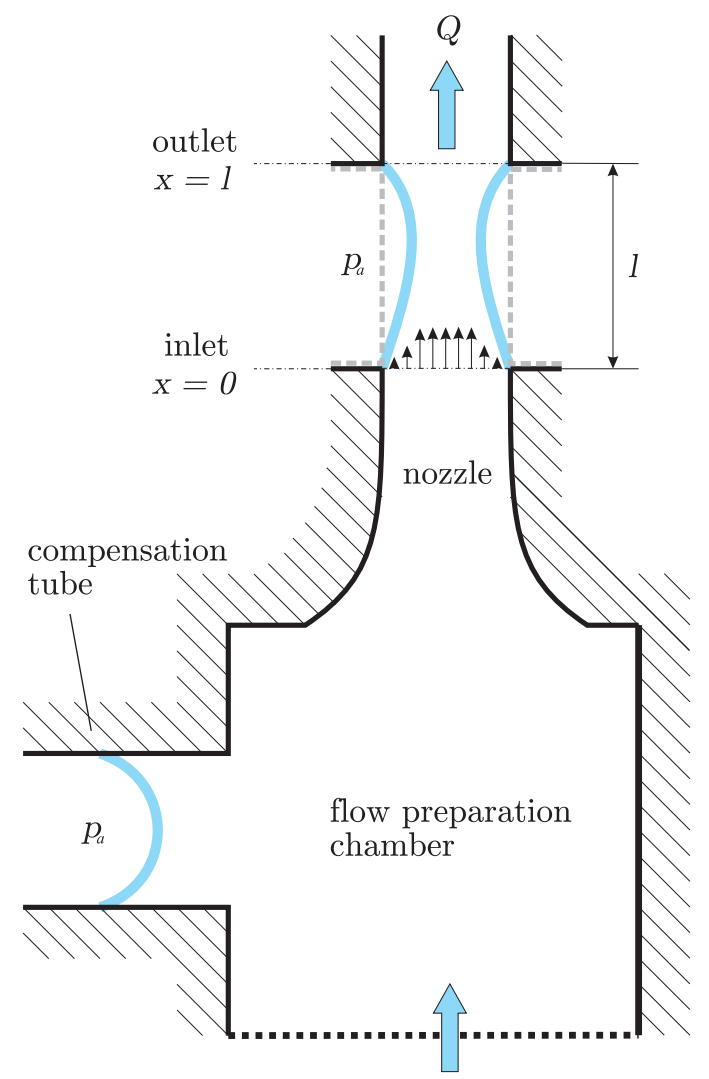

FIG. 3. (Color online) Schematic of the flow configuration and boundary conditions of the CCF ISS experiment. A reference pressure is provided by the meniscus in the compensation tube, the nozzle delivers the flow conditions at $x=0$, and the flow rate $Q$ sets the mean velocity at the outlet.

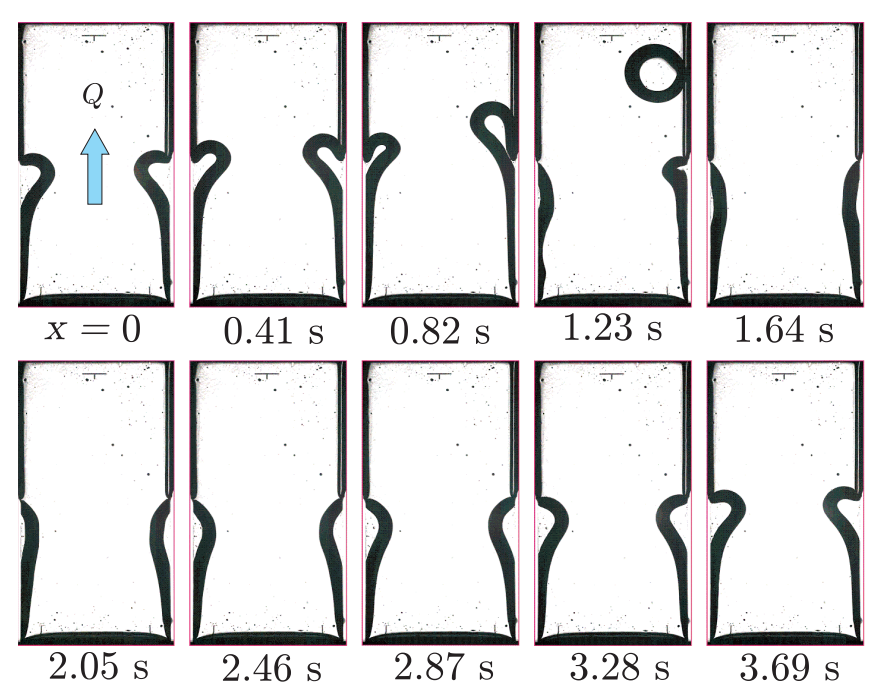

FIG. 4. (Color online) Choking phenomenon with periodical gas ingestion in a supercritical capillary channel flow (flow is upward). The free surfaces are depicted by bold curved lines in the lower half of each image. In this example $l=25 \mathrm{~mm}$ and the flow rate $Q=5.72 \mathrm{ml} / \mathrm{s}$ is $1 \%$ above the critical value. The time step between two frames is $0.41 \mathrm{~s}$ and about one bubbles in $4 \mathrm{~s}$ is ingested into the channel. pump. But as the flow rate exceeds a critical value, the interface motion and flow become unsteady as is the case in Fig. 4. In such situations the capillary pressure is too weak to prevent a temporary collapse of the free surfaces which are drawn inward, leading to gas ingestion and what is referred to herein as the choking phenomenon. This phenomenon is analogous to compressible gas flows through nozzles as velocities approach the local speed of sound [32], when elastic tubes collapse with local decreases in pressure [33], or in magnetohydrodynamic flows in what is referred to as the electromagnetic pinch effect [34]. In all these examples, a flow path is substantially constricted by the flow itself. It is a stability phenomenon because it happens rather suddenly when a critical threshold value is exceeded. The flow is either stable or unstable and thus can easily be identified by observation. However, choking in capillary channel flows does not lead to a complete severing of the flow path as occurs for forced or falling liquid jets and columns due to the Rayleigh-Plateau instability [7]. Instead, the free surfaces deflect increasingly inward, creating fingerlike intrusions that eventually pinch off ingesting bubbles into the flow. Once a bubble is ingested, the process periodically repeats, as seen in Fig. 4. In the reduced gravitational environments of spacecraft, such bubbles enter the flow path and convect downstream, where they are capable of degrading system performance by any number of means, such as by displacing liquid, plugging passageways, and stalling pumps to name a few. This makes the control of bubbles a high priority in such applications [35]. Such ingested bubbles may or may not pose a threat to terrestrial systems such as microfluidic devices, but they are certainly easier to anticipate and manage in a strong, persistent gravitational environment.

\section{MODELING THE FREE SURFACE}

A string of assumptions reduces the three-dimensional visco-inertial-capillary flow equations to a single equation that describes the predominantly one-dimensional flow and predicts the free surface contour along the channel at steady subcritical flow conditions $[6,25]$. The essential elements of the model are depicted in Fig. 3 with further details provided in the appendix. The region of interest is the capillary channel between the inlet and outlet identified in Fig. 3. In this region the steady model reduces to a simplified nonlinear momentum equation in the $x$ direction such that $\rho u d u / d x=$ $-d p / d x+d w_{f} / d x$, where terms left to right represent convective acceleration, channel capillary pressure gradient, and viscous resistance, respectively. The local pressure in the channel is governed by the curvature of the free surface. In the "no flow" state, the pressure in the channel is determined solely by the capillary meniscus curvature in the compensation tube as identified in Fig. 3. For nonzero velocities the pressure decreases within the channel due to convective acceleration and viscous resistance $w_{f}$. Considering the steady flow of an incompressible liquid, the flow rate $Q=u A$ is constant, where $A=A(x)$ is the flow cross section. The smaller the cross section $A$, the higher the mean flow velocity $u$. The pressure decreases along the channel due to $w_{f}$. The momentum loss rate is constant for a fully developed flow but varies along the channel when the flow is developing, as is the case for the CCF experiment. Developing flows possess boundary layers with large velocity gradients and accompanying increases in $w_{f}$. 
Our governing equation requires two boundary conditions. The first is given by the constant pressure $p_{0}$ at the channel inlet, where $p_{0}$ is the sum of the no-flow static pressure and the pressure losses due to viscous friction and convective acceleration within the entrance nozzle. While the static pressure is defined by the capillary meniscus pressure in the compensation tube, the flow rate-dependent terms are complicated by the flow path geometry requiring full numerical computations to assess the inlet pressure boundary condition for the otherwise analytic channel flow problem. The second boundary condition requires knowledge of the velocity distribution at the channel inlet. In this way $w_{f}(x=0)$ is known. But the entrance flow cannot be described analytically for this combined nozzle-channel conduit and we instead opt to model the channel entrance velocity using the two limiting cases of a fully developed Poiseuille flow [32] and a uniform plug flow. For the plug flow inlet assumption an analytical solution for the subsequent developing entrance flow is available for the parallel plate geometry [36]. Once both boundary conditions are known, the free surface contour can be found by solving the model equation numerically using a finite difference method [37]. The critical flow rate is identified using a Newton iteration scheme between subcritical conditions that can be computed and supercritical conditions that cannot.

\section{RESULTS}

\section{A. Critical free surface profiles}

Figure 5 shows three surface contours typical of these CCF ISS experiments. Each interface contour corresponds to the last stable surface just before choking occurs. Our model predictions are provided for comparison. As identified in Fig. 2, $k$ denotes the distance of the free surface to the channel centerline. The free surface is pinned at $k=12.5 \mathrm{~mm}$ at both the inlet $x=0$ and outlet $x=l$, as shown in Fig. 3 . In all cases, the surfaces bend inwards to form a neck where $k \approx 10 \mathrm{~mm}$ and where the surface first becomes unstable. The highly simplified model proves as adequate tool to predict the free surface at subcritical flow rates. As can be seen in Fig. 5, the predictions of both limiting inlet conditions, i.e., plug flow and Poiseuille flow, practically coincide. Nonetheless, deviations between experiments and predictions are evident. Both the experiments as well as three-dimensional numerical simulations have confirmed that the neck region generates a wake leading to downstream separation and a clear violation of the laminar flow assumption.

\section{B. Critical flow rate}

Figure 6 presents the critical flow rate for the accessible range of channel lengths for these experiments. In contrast to previous experiments where the channel length was fixed, the slider surfaces of the CCF ISS experiment are used to vary the channel length. The long duration low- $g$ environment $\sim 10^{-5} g_{o}$ aboard ISS [38] provides ample time to sweep the flow domain and determine accurate critical flow rates. It shows that for long channels the experimental data agree well with the predictions. Here, the measured values are bracketed by the limiting cases of Poiseuille and plug flow between which the real inlet flow condition is expected. For shorter
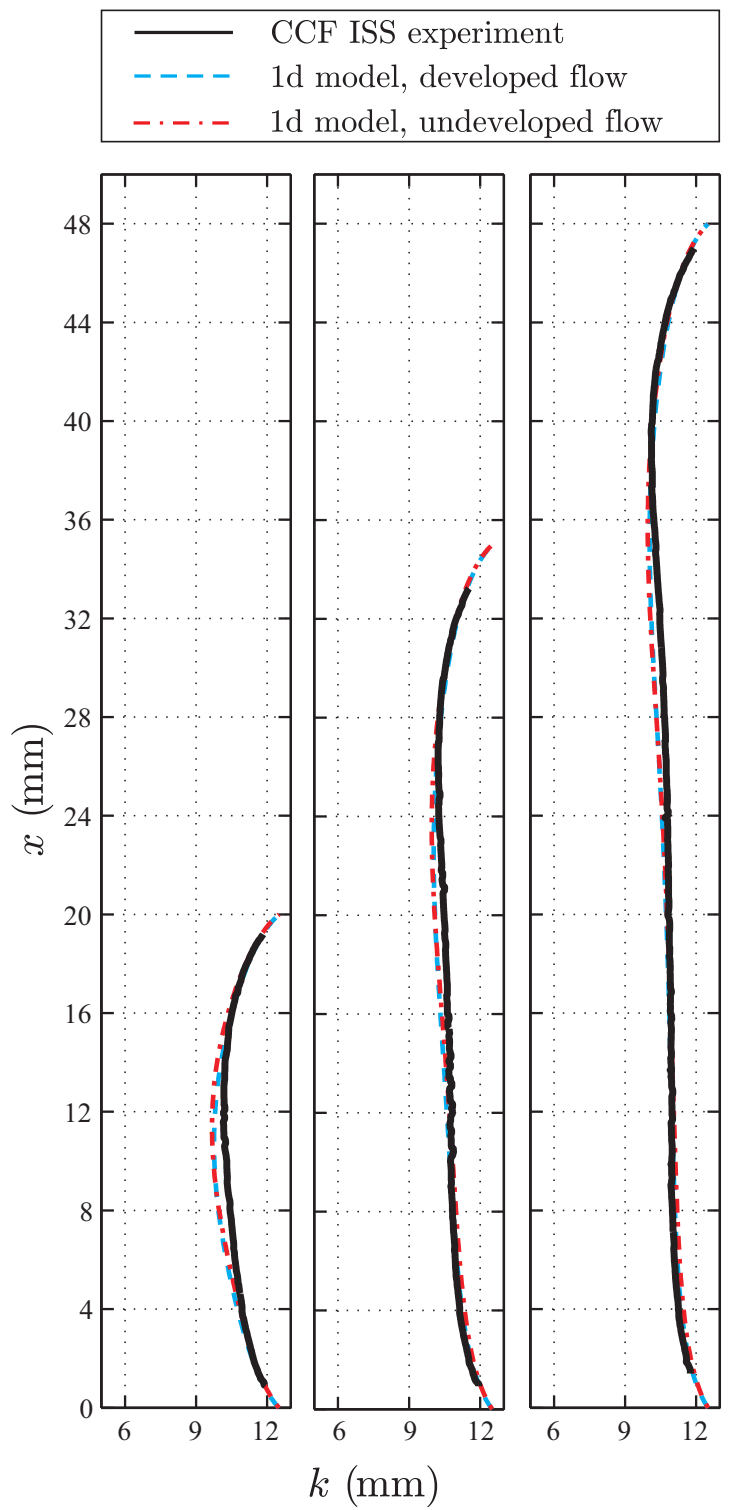

FIG. 5. (Color online) Three representative free surface contours for parallel plate channels of different lengths for the maximum flow rate with stable deformations. The combined systematic and statistic error of the contour position $k(x)$ due to flow rate adjustment and image processing is below $0.2 \mathrm{~mm}$. The critical flow rates in experiment, model with plug inflow, and model with Poiseuille inflow were (i) for $l=20 \mathrm{~mm}: Q_{c}=5.87,5.84,6.29 \mathrm{ml} / \mathrm{s}$, (ii) for $l=35 \mathrm{~mm}: Q_{c}=5.55,5.31,5.82 \mathrm{ml} / \mathrm{s}$, and (iii) for $l=48 \mathrm{~mm}$ : $Q_{c}=5.27,5.06,5.58 \mathrm{ml} / \mathrm{s}$. Further increases of the flow rate would lead to choking as shown in Fig. 4.

channels, however, there is considerable deviation where the model overestimates the critical flow rate by underpredicting pressure losses. Qualitative agreement is preserved despite the increasing violation of the laminar flow assumption.

\section{CONCLUSIONS AND OUTLOOK}

Experimental data for an extended parameter range inaccessible to terrestrial research was collected using the Capillary Channel Flow experiment aboard the International Space Station (CCF ISS). One of the primary experiment objectives 


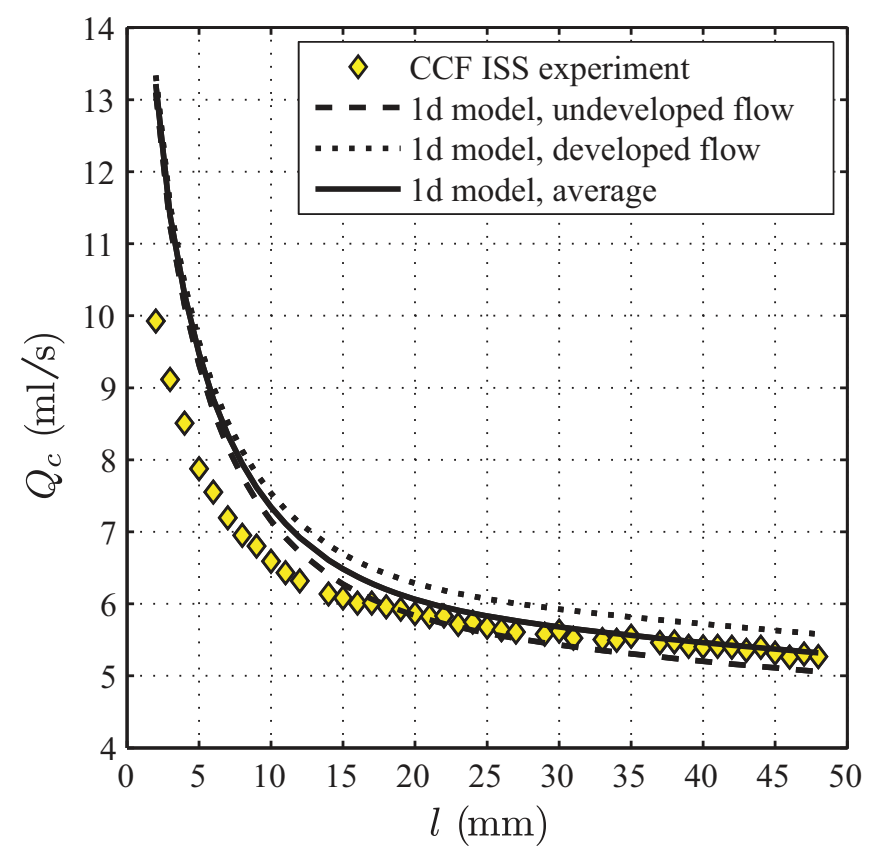

FIG. 6. (Color online) Dependence of critical flow rate on open channel length for both experimental results and numerical predictions for the parallel plate channel. The experimental error is less than $\pm 0.2 \mathrm{ml} / \mathrm{s}$.

of the effort is reported here concerning the prediction of dynamic surface contours and critical flow rates between a partially open channel consisting of two parallel plates, to which the applicability of a simplified model is demonstrated. Significantly more CCF ISS data have been and are being collected on other aspects of the flow, such as supercritical flow phenomena with gas ingestion, critical transient flows, and all flow types in the groove and wedge channel geometries (Fig. 1). Bubbly two-phase flows in the wedge channel are also studied at length, with attention paid to interface stabilization via bubble injection, bubble mergers, free surface coalescence, and conditions where such conduits perform the passive function of bubbly-flow phase separations in a low acceleration environment. The CCF experiment hardware is currently residing on the ISS and further experiments are scheduled in the near future.

\section{ACKNOWLEDGMENTS}

L. Kiewidt contributed to the CCF data collection and image processing. Y. Chen, R. Jenson, and W. Blackmore also contributed to the data collection. The authors acknowledge the support provided by the NASA cadre at Marshall Space Flight Center and astronauts Scott Kelly, Catherine Coleman, and Mike Fossum. Thanks are due to the technical staff at Astrium for manufacturing the experiment hardware and for technical support during the experiments. The German research team acknowledges support through an award from the NASA Research Announcement NRA-94-OLMSA-05 and is currently supported financially by the German Federal Ministry of Economics and Technology (BMWi) via the German Aerospace Center (DLR) under Grant No. 50WM1145. The U.S. research team acknowledges support through an award from the NASA Research Announcement Microgravity Fluid Physics: Research and Flight Experiment Opportunities (NRA-98HEDS-03) and is currently supported in part under NASA cooperative agreement NNX12AO47A.

\section{APPENDIX: ONE-DIMENSIONAL MODEL OF FREE SURFACE PROFILE}

The flow is assumed to be steady, incompressible, isothermal, laminar, and one dimensional with a mean velocity $u$ in the $x$ direction. The open channel is connected to closed ducts at both the inlet and outlet. For these conditions, the conservation of momentum reads

$$
\underbrace{\rho u \frac{d u}{d x}}_{\text {convective acceleration }}=-\underbrace{\frac{d p}{d x}}_{\text {pressure }}+\underbrace{\frac{d w_{f}}{d x}}_{\text {irreversible pressure loss }} .
$$

\section{The convective acceleration term in Eq. (A1)}

The liquid is incompressible, therefore it follows from flow continuity that the flow rate $Q=u A$ is constant along the channel, i.e., in the $x$ direction. It is

$$
u \frac{d A}{d x}+A \frac{d u}{d x}=0,
$$

where $u(x)$ is the average velocity and $A(x)$ the flow cross section along the channel. Rearranged, Eq. (A2) gives

$$
u \frac{d u}{d x}=-\frac{Q^{2}}{A^{3}} \frac{d A}{d x},
$$

which can be inserted into the momentum conservation Eq. (A1).

\section{The pressure in Eq. (A1)}

When the liquid is flowing, the pressure $p$ also varies along the channel and is dictated by the ambient pressure $p_{a}$ and the pressure jump at the free surface due to surface tension $\sigma$ and surface curvature $h$. This is expressed by the Young-Laplace equation

$$
p-p_{a}=-\sigma h=-\sigma\left(\frac{1}{R_{1}}+\frac{1}{R_{2}}\right),
$$

where the curvature can also be given by the two principal radii $R_{1}$ and $R_{2}$ on each point of the surface. Viewed along the surface contour $k(x)$, which is shown in the lower picture of Fig. 2, the first principal radius of curvature $R_{1}(x)$ lies in the plane orthogonal to this direction of view. We assume that the two free surfaces are mirror symmetric with respect to $z=0$ and restrict our considerations to positive $k(x)$ at the region with $z>0$. If the surface contour has a slope of zero, $R_{1}$ lies in the cross-sectional $y z$ plane of the channel; see the upper picture of Fig. 2. The second principal radius of curvature is applied along the channels $x z$ center plane; see the lower picture of Fig. 2. The first derivative of pressure used in Eq. (A1) reads

$$
\frac{d p}{d x}=-\sigma \frac{d h}{d x}=-\sigma \frac{d}{d x}\left(\frac{1}{R_{1}}+\frac{1}{R_{2}}\right) .
$$


Not only curvature but also the flow cross section $A$ is connected to $R$ (see upper picture of Fig. 2). Due to the slope $d_{x} k=d k / d x$ of the free surface along the channel, the $R$ in Fig. 2 is not identical to $R_{1}$. Two cases are distinguished: (i) the contact line is pinned along the edge of the plate so $k \leqslant b / 2-a / 2$ and (ii) the contact line is not pinned and free to move with a zero degree contact angle so $k>b / 2-a / 2$. The flow cross-sectional area is

$$
A(k)= \begin{cases}a b+a\left(k+R-\frac{b}{2}\right)-2 R^{2} \arcsin \left(\frac{a}{2 R}\right) & \text { for } \quad k \geqslant \frac{b}{2}-\frac{a}{2} \\ a^{2}+2 a k-\frac{\pi}{4} a^{2} & \text { for } \quad k<\frac{b}{2}-\frac{a}{2}\end{cases}
$$

with the radius $R$ being

$$
R(k)=\left\{\begin{array}{ll}
\frac{a^{2}+(b-2 k)^{2}}{4(b-2 k)} & \text { for } k \geqslant \frac{b}{2}-\frac{a}{2} \\
\frac{a}{2} & \text { for } k<\frac{b}{2}-\frac{a}{2}
\end{array} .\right.
$$

The first principal radius of curvature becomes

$$
R_{1}= \begin{cases}\frac{a^{2}+(b-2 k)^{2}\left(1+\left(d_{x} k\right)^{2}\right)}{4(b-2 k)\left(1+\left(d_{x} k\right)^{2}\right)^{1 / 2}} & \text { for } \quad k \geqslant \frac{b}{2}-\frac{a}{2\left(1+\left(d_{x} k\right)^{2}\right)^{1 / 2}} \\ \frac{a}{2} & \text { for } k<\frac{b}{2}-\frac{a}{2\left(1+\left(d_{x} k\right)^{2}\right)^{1 / 2}}\end{cases}
$$

with a difference between the range of $k$ here at $R_{1}$ and in the cross-sectional area $A$ due to the slope $d_{x} k$ of the free surface. The second principal radius of curvature can be derived from the surface contour $k(x)$ in the $x z$ plane and becomes

$$
R_{2}=\frac{\left(1+\left(d_{x} k\right)^{2}\right)^{\frac{3}{2}}}{d_{x}^{2} k} \text {. }
$$

\section{The irreversible pressure loss in Eq. (A1)}

The irreversible pressure loss depends on the geometry and boundaries of the flow path but also on whether the flow is yet developed. In general, this work consumed by friction $w_{f}$ contains one component for the fully developed Poiseulle flow $w_{\text {Pf }}$ and another component for the entrance region flow that can be modeled with an approach of Sparrow [36] denoted $w_{\text {Sf. Hence, }}$

$$
w_{f}=w_{\mathrm{Pf}}+w_{\mathrm{Sf}} .
$$

Figure 3 gives an impression on the flow conditions in the CCF ISS experiment. Coming from a liquid reservoir, there is a rectangular duct flow through a nozzle, then the channel flow between the parallel plates, which is the region of interest, followed by a duct flow again. And, indeed, the flow in the channel is not fully developed but still part of the entrance region of the flow. A law for the fully developed flow component can be found in fluid mechanics textbooks like [32] and is

$$
w_{\mathrm{Pf}}=\frac{\rho}{2} u^{2} \frac{K_{\mathrm{Pf}}}{\operatorname{Re} D_{h}} x
$$

or

$$
\frac{d w_{\mathrm{Pf}}}{d x}=\frac{\rho}{2} u^{2} \frac{K_{\mathrm{Pf}}}{\operatorname{Re} D_{h}},
$$

with $D_{h}$ being the hydraulic diameter (4 times the flow cross section divided by the wetted perimeter) and $K_{\mathrm{Pf}}$ being a configuration-dependent coefficient. In the parallel plates geometry, see Ref. [32],

$$
D_{h}=2 a, \quad K_{\mathrm{Pf}}=96
$$

and the Reynolds number $\operatorname{Re}$ is

$$
\operatorname{Re}=\frac{u D_{h}}{v} .
$$

Incorporating the effect of the entrance region in a duct of constant cross section, Sparrow [36] finds

$$
w_{f}=\frac{\rho}{2} u^{2} \frac{1}{\operatorname{Re} D_{h}}\left(K_{\mathrm{Pf}} x+16 K_{\mathrm{Sf}}(x)\right)
$$

or

$$
\frac{d w_{f}}{d x}=\frac{\rho}{2} u^{2} \frac{1}{\operatorname{Re} D_{h}}\left(K_{\mathrm{Pf}}+16 \hat{K}_{\mathrm{Sf}}(x)\right),
$$

where the coefficient $K_{\text {Sf }}$ depends on the length $x$ along which the flow develops and $\hat{K}_{\mathrm{Sf}}$ is the gradient of $K_{\mathrm{Sf}}$ in the $x$ direction. This length $x$ is transformed into a scaled length $\hat{x}$ as

$$
\hat{x}=\frac{16 x}{\operatorname{Re} D_{h}},
$$

for which the dependence of $K_{\mathrm{Sf}}$ is formulated. For the CCF model, Sparrows solution in terms of infinite rows is not applied in its original form but approximated by a more convenient expression. It is found that

$$
K_{\mathrm{Sf}}(\hat{x})=c_{0}+c_{1} \exp \left(c_{2} \hat{x}^{c_{3}}\right)
$$

and

$$
\hat{K}_{\mathrm{Sf}}(\hat{x})=c_{1} c_{2} c_{3} \exp \left(c_{2} \hat{x}^{c_{3}}\right) \hat{x}^{c_{3}-1}
$$




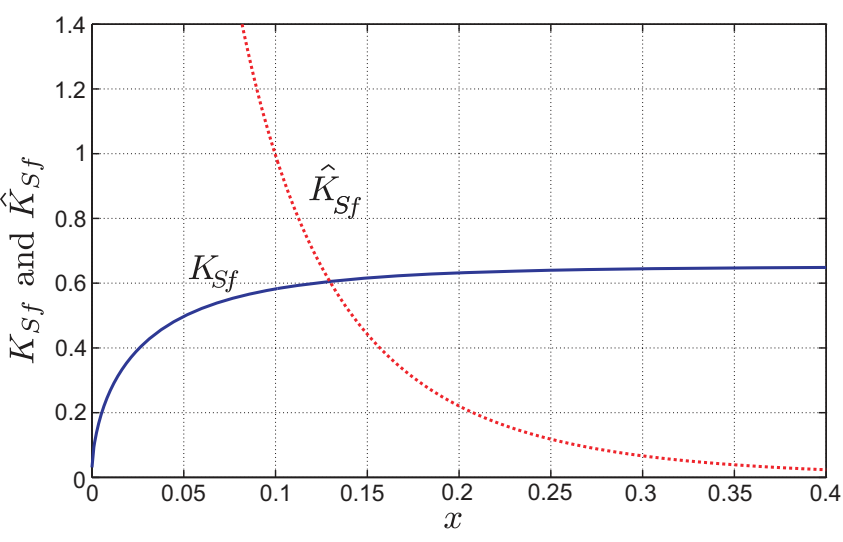

FIG. 7. (Color online) Pressure loss factor $K_{\mathrm{Sf}}$ and its gradient $\hat{K}_{\mathrm{Sf}}$ in dependence of the transformed entrance length $\hat{x}$ for the entrance flow between two parallel plates.

with the constant factors $c_{0}=0.6511, c_{1}=-0.6203, c_{2}=$ -9.9948 , and $c_{3}=0.6576$. Figure 7 shows the trends of both $K_{\text {Sf }}$ and $\hat{K}_{\text {Sf }}$.

\section{Boundary conditions of Eq. (A1)}

At the inlet at $x=0$ and the outlet at $x=l$, respectively, the boundary conditions read

$x=0: A_{0}=a b ; \quad k=b / 2 ; \quad u_{0}=Q / A_{0} ; \quad p=p_{0} ; \quad \hat{x}$

$x=l: A_{l}=A_{0} ; \quad k=b / 2 ; \quad u_{l}=u_{0}$.

The two last boundary conditions at $x=0$ require special attention. The first one is the pressure $p_{0}$ at the inlet, and the second is how developed the flow already is which is expressed by a the transformed flow development length $\hat{x}$.

\section{Boundary condition inlet pressure}

The contour of the free surface depends on the pressure jump across the free surface, and therefore the initial pressure $p_{0}$ at the channel inlet at $x=0$ is a necessary boundary condition. For a given system such as the CCF ISS experimental setup, the pressure loss along the flow path between a reference location with known pressure and the channel inlet has to be evaluated with numerical simulations. The reference location with known pressure is the compensation tube with a meniscus of given curvature. Due to viscous losses along the flow path, a part $p_{0} \propto Q$ is expected. And due to convective acceleration, a part $p_{0} \propto Q^{2}$ is expected. Accordingly, a relation for the pressure difference across the interface at the channel inlet is found to be

$$
p_{0}-p_{a}=\frac{2 \sigma}{R_{\mathrm{CT}}}+K_{1} \frac{\mu}{2 D_{h} A_{0}} Q+K_{2} \frac{\rho}{2 A_{0}^{2}} Q^{2},
$$

with $R_{\mathrm{CT}}=30 \mathrm{~mm}$ being the radius of the compensation tube and, from numerical simulations, $K_{1}=527$ and $K_{2}=1.68$.

\section{Boundary condition inlet flow profile}

The degree of flow development at the channel inlet determines the irreversible pressure loss $d w_{f} / d x$ along the channel. However, the channel is fed through a rectangular duct nozzle with a variable cross section which cannot be described analytically. We pose therefore as two possible inlet conditions (i) undeveloped flow, i.e., $\hat{x}=0$, and (ii) fully developed flow, i.e., $\hat{x} \geqslant 0.24$, leading to $K_{S f}=0$ in Eqs. (A19) and (A16).
[1] T. Gambaryan-Roisman and P. Stephan, J. Heat Transf. 131, 033101 (2009).

[2] B. V. Kichatov, A. P. Mikhalev, and V. M. Polyaev, High Temp. 38, 460 (2000).

[3] B. J. Lowry and D. B. Thiessen, Phys. Fluids 19, 022102 (2007).

[4] O. A. Kabov, M. V. Bartashevich, and V. Cheverda, Int. J. Emerg. Multidisc. Fluid Sc. 2, 161 (2010).

[5] J. Melin, W. Wijngaart, and G. Stemme, Lab Chip 5, 682 (2005).

[6] U. Rosendahl, A. Ohlhoff, and M. E. Dreyer, J. Fluid Mech. 518, 187 (2004).

[7] J. Eggers, Rev. Mod. Phys. 69, 865 (1997).

[8] J. Kern, Verfahrenstechnik 3, 425 (1969).

[9] C. A. Perazzo and J. Gratton, J. Fluid Mech. 507, 367 (2004).

[10] K. Mertens, V. Putkaradze, and P. Vorobieff, J. Fluid Mech. 531, 49 (2005)

[11] N. LeGrand-Piteira, A. Daerr, and L. Limat, Phys. Rev. Lett. 96, 254503 (2006).

[12] B. Birnir, K. Mertens, V. Putkaradze, and P. Vorobieff, Phys. Rev. Lett. 101, 114501 (2008).

[13] R. Siegel, J. Appl. Mech. 83, 165 (1961).

[14] S. Levine et al., J. Colloid Interf. Sci. 73, 136 (1980).

[15] M. E. Dreyer, A. Delgado, and H. J. Rath, J. Colloid Interf. Sci. 183, 158 (1994).
[16] J. R. Rollins, R. K. Grove, and D. E. J. Jaekle, in Proceedings of the 21 st AIAA Joint Propulsion Conference (AIAA, Monterey, California, USA, 1985), p. 1.

[17] D. E. J. Jaekle, in Proceedings of the 27th AIAA/SAE/ASME/ASEE Joint Propulsion Conference (AIAA, Sacramento, California, USA, 1991), p. 1.

[18] K. Kinefuchi, J. Spacecr. Rockets 47, 860 (2010).

[19] M. E. Dreyer et al., Space Forum 3, 87 (1998).

[20] B. Motil et al., in Proceedings of the Conference and Exhibit on International Space Station Utilization (AIAA, Cape Canaveral, Florida, USA, 2001).

[21] U. Rosendahl, C. Fechtmann, and M. E. Dreyer, in Proceedings of the 17th ESA Symposium on European Rocket and Balloon Programmes and Related Research (ESA, Sandefjord, Norway, 2005), p. 551.

[22] U. Rosendahl, A. Grah, and M. E. Dreyer, Phys. Fluids 22, 052102, 1 (2010).

[23] A. Salim, C. Colin, and M. E. Dreyer, Microgravity Sci. Technol. 22, 87 (2010).

[24] A. Salim et al., Int. J. Multiphas. Flow 36, 707 (2010).

[25] A. Grah et al., J. Fluid Mech. 600, 271 (2008).

[26] A. Grah and M. E. Dreyer, Phys. Fluids 22, 014101 (2010).

[27] D. Haake et al., Ann. N.Y. Acad. Sci. 1077, 443 (2006). 
[28] D. Haake et al., Microgravity Sci. Technol. 22, 129 (2010).

[29] J. Klatte, Ph.D. thesis, University of Bremen, 2011.

[30] R. A. Spivey, W. A. Sheredy, and G. Flores, in Proceedings of the 46th AIAA Aerospace Sciences Meeting and Exhibit (AIAA, Reno, Nevada, USA, 2008), p. 1.

[31] P. J. Canfield et al., Exp. Fluids 54, 1519 (2013).

[32] F. M. White, Fluid Mechanics, 5th ed. (McGraw Hill, New York, 2003)

[33] J. B. Grotberg and O. E. Jensen, Annu. Rev. Fluid Mech. 36, 121 (2004).
[34] J. U. Mohring, C. Karcher, and D. Schulze, Phys. Rev. E 71, 047301 (2005).

[35] National Research Council, Recapturing a Future for Space Exploration (National Academic Press, Washington DC, 2011).

[36] E. M. Sparrow, S. H. Lin, and T. S. Lundgren, Phys. Fluids 7, 338 (1964).

[37] E. Kreyszig, Advanced Engineering Mathematics, 9th ed. (John Wiley \& Sons, New York, 2006).

[38] E. S. Nelson and K. Jules, J. Gravi. Physiol. 11, 1 (2004). 